\title{
ABSOLUTELY CONTINUOUS MEASURES AND COMPACT COMPOSITION OPERATOR ON SPACES OF CAUCHY TRANSFORMS
}

\author{
YUSUF ABU MUHANNA and EL-BACHIR YALLAOUI
}

Received 5 October 2003

\begin{abstract}
The analytic self-map of the unit disk $\mathbf{D}, \varphi$ is said to induce a composition operator $C_{\varphi}$ from the Banach space $X$ to the Banach space $Y$ if $C_{\varphi}(f)=f \circ \varphi \in Y$ for all $f \in X$. For $z \in \mathbf{D}$ and $\alpha>0$, the families of weighted Cauchy transforms $F_{\alpha}$ are defined by $f(z)=\int_{\mathrm{T}} K_{x}^{\alpha}(z) d \mu(x)$, where $\mu(x)$ is complex Borel measure, $x$ belongs to the unit circle T, and the kernel $K_{x}(z)=$ $(1-\bar{x} z)^{-1}$. In this paper, we will explore the relationship between the compactness of the composition operator $C_{\varphi}$ acting on $F_{\alpha}$ and the complex Borel measures $\mu(x)$.
\end{abstract}

2000 Mathematics Subject Classification: 30E20, 30D99.

1. Background. Let $\mathbf{T}$ be the unit circle and $\mathbf{M}$ the set of all complex-valued Borel measures on $\mathbf{T}$. For $\alpha>0$ and $z \in \mathbf{D}$, we define the space of weighted Cauchy transforms $F_{\alpha}$ to be the family of all functions $f(z)$ such that

$$
f(z)=\int_{\mathrm{T}} K_{x}^{\alpha}(z) d \mu(x),
$$

where the Cauchy kernel $K_{x}(z)$ is given by

$$
K_{x}(z)=\frac{1}{1-\bar{x} z}
$$

and where $\mu$ in (1.1) varies over all measures in $\mathbf{M}$. The class $F_{\alpha}$ is a Banach space with respect to the norm

$$
\|f\|_{F_{\alpha}}=\inf \|\mu\|_{\mathrm{M}}
$$

where the infimum is taken over all Borel measures $\mu$ satisfying (1.1). $\|\mu\|$ denotes the total variation norm of $\mu$. The family $F_{1}$ has been studied extensively in the Soviet literature. The generalizations for $\alpha>0$ were defined by MacGregor [7]. The Banach spaces $F_{\alpha}$ have been well studied in [2, 3, 5, 7]. Among the properties of $F_{\alpha}$ we list the following:

(i) $F_{\alpha} \subset F_{\beta}$ whenever $0<\alpha<\beta$,

(ii) $F_{\alpha}$ is Möbius invariant,

(iii) $f \in F_{\alpha}$ if and only if $f^{\prime} \in F_{1+\alpha}$ and $\left\|f^{\prime}\right\|_{F_{1+\alpha}} \leq \alpha\|f\|_{F_{\alpha}}$,

(iv) if $g \in F_{\alpha+1}$, then $f(z)=\int_{0}^{z} g(w) d w \in F_{\alpha}$ and $\|f\|_{F_{\alpha}} \leq(2 / \alpha)\|g\|_{F_{1+\alpha}}$.

The space $F_{\alpha}$ may be identified with $\mathbf{M} / \overline{H_{0}^{1}}$, the quotient of the Banach space $\mathbf{M}$ of Borel measures by $\overline{H_{0}^{1}}$, the subspace of $L^{1}$ consisting of functions with mean value zero 
whose conjugate belongs to the Hardy space $H^{1}$. Hence, $F_{\alpha}$ is isometrically isomorphic to $\mathbf{M} / \overline{H_{0}^{1}}$. Furthermore, $\mathbf{M}$ admits a decomposition $\mathbf{M}=L^{1} \oplus \mathbf{M}_{s}$, where $\mathbf{M}_{s}$ is the space of Borel measures, which are singular with respect to Lebesgue measure, and $\overline{H_{0}^{1}} \subset L^{1}$. According to the Lebesgue decomposition theorem, any $\mu \in \mathbf{M}$ can be written as $\mu=$ $\mu_{a}+\mu_{s}$, where $\mu_{a}$ is absolutely continuous with respect to the Lebesgue measure and $\mu_{s}$ is singular with respect to the Lebesgue measure $\left(\mu_{a} \perp \mu_{s}\right)$. Since $|x|=1$ in (1.1), if we let $x=e^{i t}$, then we can decompose $\mu$ in such a way that $d \mu\left(e^{i t}\right)=h\left(e^{i t}\right) d t+d \mu_{s}\left(e^{i t}\right)$, where $h\left(e^{i t}\right) \in L^{1}$. Consequently, $F_{\alpha}$ is isomorphic to $L^{1} / \overline{H_{0}^{1}} \oplus \mathbf{M}_{s}$. Hence, $F_{\alpha}$ can be written as $F_{\alpha}=F_{\alpha a} \oplus F_{\alpha s}$, where $F_{\alpha a}$ is isomorphic to $L^{1} / \overline{H_{0}^{1}}$, the closed subspace of $\mathbf{M}$ of absolutely continuous measures, and $F_{\alpha s}$ is isomorphic to $\mathbf{M}_{s}$, the subspace of $\mathbf{M}$ of singular measures. If $f \in F_{\alpha a}$, then the singular part is null and the measure $\mu$ for which (1.1) holds reduces to $d \mu(t)=d \mu\left(e^{i t}\right)=h\left(e^{i t}\right) d t$, where $h\left(e^{i t}\right) \in L^{1}$ and $d t$ is the Lebesgue measure on $\mathrm{T}$, see [1]. The functions in $F_{\alpha a}$ may be then written as, for $x=e^{i t}$,

$$
f(z)=\int_{-\pi}^{\pi} K_{x}^{\alpha}(z) h\left(e^{i t}\right) d t .
$$

Furthermore, if $h\left(e^{i t}\right)$ is nonnegative, then

$$
\|f\|_{F_{\alpha}}=\left\|h\left(e^{i t}\right)\right\|_{L^{1}} .
$$

REMARK 1.1. For simplicity, we will adopt the following notation throughout the paper. We will reserve $\mu$ for the Borel measures of $\mathbf{M}$, and since in (1.1) $|x|=1$, we can write $x=e^{i t}$, where $t \in[-\pi, \pi)$. We will reserve $d t$ for the normalized Lebesgue of the unit circle $\mathbf{T}$, and $d \sigma$ for the singular part of $d \mu$. Hence, instead of writing $d \mu\left(e^{i t}\right)=$ $d \mu_{a}\left(e^{i t}\right)+d \mu_{s}\left(e^{i t}\right)=h\left(e^{i t}\right) d t+d \mu_{s}\left(e^{i t}\right)$, we may simply write $d \mu(t)=h d t+d \sigma(t)$.

2. Introduction. If $X$ and $Y$ are Banach spaces, and $L$ is a linear operator from $X$ to $Y$, we say that $L$ is bounded if there exists a positive constant $A$ such that $\|L(f)\|_{Y} \leq A\|f\|_{X}$ for all $f$ in $X$. We denote by $C(X, Y)$ the set of all bounded linear operators from $X$ to $Y$. If $L \in C(X, Y)$, we say that $L$ is a compact operator from $X$ to $Y$ if the image of every bounded set of $X$ is relatively compact (i.e., has compact closure) in $Y$. Equivalently, a linear operator $L$ is a compact operator from $X$ to $Y$ if and only if for every bounded sequence $\left\{f_{n}\right\}$ of $X,\left\{L\left(f_{n}\right)\right\}$ has a convergent subsequence in $Y$. We will denote by $K(X, Y)$ the subset of $C(X, Y)$ of compact linear operators from $X$ into $Y$.

Let $H(\mathbf{D})$ denote the set of all analytic functions on the unit disk $\mathbf{D}$ and map $\mathbf{D}$ into D. If $X$ and $Y$ are Banach spaces of functions on the unit disk D, we say that $\varphi \in H(\mathbf{D})$ induces a bounded composition operator $C_{\varphi}(f)=f(\varphi)$ from $X$ to $Y$, if $C_{\varphi} \in C(X, Y)$ or equivalently $C_{\varphi}(X) \subseteq Y$, and there exists a positive constant $A$ for all $f \in X$ and $\left\|C_{\varphi}(f)\right\|_{Y} \leq A\|f\|_{X}$. In case $X=Y$, then we say that $\varphi$ induces a composition operator $C_{\varphi}$ on $X$. If $f \in X$, then $C_{\varphi}(f)=f(\varphi) \in X$. Similarly, we say that $\varphi \in H(\mathbf{D})$ induces a compact composition operator if $C_{\varphi} \in K(X, Y)$.

A fundamental problem that has been studied concerning composition operators is to relate function theoretic properties of $\varphi$ to operator theoretic properties of the restriction of $C_{\varphi}$ to various Banach spaces of analytic functions. However, since the 
spaces of Cauchy transforms are defined in terms of Borel measures, it seems natural to investigate the relation between the behavior of the composition operator and the measure. The work in this paper was motivated by the work of Cima and Matheson in [1], who showed that $C_{\varphi}$ is compact on $F_{1}$ if and only if $C_{\varphi}\left(F_{1}\right) \subset F_{1 a}$. In our work, we will generalize this result for $\alpha>1$.

Now, if $C_{\varphi} \in C\left(F_{\alpha}, F_{\alpha}\right)$, then $C_{\varphi}(f)=(f \circ \varphi)=f(\varphi) \in F_{\alpha}$ for all $f \in F_{\alpha}$ and there exists a positive constant $A$ such that

$$
\left\|C_{\varphi}(f)\right\|_{F_{\alpha}}=\|f(\varphi)\|_{F_{\alpha}}=\|[\mu]\| \leq A\|f\|_{F_{\alpha}} .
$$

Since $F_{\alpha}$ can be identified with the quotient space $\mathbf{M} / \overline{H_{0}^{1}}$, we can view $C_{\varphi}$ as a map,

$$
\begin{aligned}
C_{\varphi}: \mathbf{M} / \overline{H_{0}^{1}} & \longrightarrow \mathbf{M} / \overline{H_{0}^{1}}, \\
f & \longmapsto f(\varphi) .
\end{aligned}
$$

The equivalence class of a complex measure $\mu$ will be written as

$$
\begin{gathered}
{[\mu]=\mu+\overline{H_{0}^{1}}=\left\{\mu+\bar{h}: h \in H_{0}^{1}\right\},} \\
\|[\mu]\|=\inf _{h}\|\mu+\bar{h}\| .
\end{gathered}
$$

The space $C\left(F_{\alpha}, F_{\alpha}\right)$ has been studied in [4], where Hibschweiler showed that

(1) if $\alpha \geq 1$, then $C_{\varphi} \in C\left(F_{\alpha}, F_{\alpha}\right)$ for any analytic self map $\varphi$ of the unit disc,

(2) $C_{\varphi} \in C\left(F_{\alpha}, F_{\alpha}\right)$ if and only if $\left\{K_{\chi}^{\alpha}(\varphi):|x|=1\right\}$ is a norm bounded subset of $F_{\alpha}$,

(3) if $C_{\varphi} \in C\left(F_{\alpha}, F_{\alpha}\right)$, then $C_{\varphi} \in C\left(F_{\beta}, F_{\beta}\right)$ for $0<\alpha<\beta$,

(4) if $C_{\varphi} \in C\left(F_{\alpha}, F_{\alpha}\right)$, then the operator $\varphi^{\prime} C_{\varphi} \in C\left(F_{\alpha+1}, F_{\alpha+1}\right)$.

In this paper, we will investigate necessary and sufficient conditions for $C_{\varphi}$ to be compact on $F_{\alpha}$ for $\alpha \geq 1$. Since $F_{\alpha}$ is Mobius invariant, then there is no loss of generality in assuming that $\varphi(0)=0$.

3. Compactness and absolutely continuous measures. In this section, we will show that the compactness of the composition operator $C_{\varphi}$ on $F_{\alpha}$ is strongly tied with the absolute continuity of the measure that supports it. First, we state this lemma due to [6].

LEMMA 3.1. If $0<\alpha<\beta$, then $F_{\alpha} \subset F_{\beta a}$ and the inclusion map is a compact operator of norm one.

Next, we show that the bounded function of $F_{\alpha}$ belongs to $F_{\alpha a}$ for $\alpha \geq 1$.

Proposition 3.2. $H^{\infty} \cap F_{\alpha} \subset F_{\alpha a}$ for $\alpha \geq 1$.

Proof. Suppose that $f \in H^{\infty} \cap F_{\alpha}$, where $\alpha \geq 1$. Then, using the previous lemma and the fact that $H^{\infty} \subset F_{1 a}$, we get that for any $z \in \mathbf{D}, f(z) \in H^{\infty} \cap F_{\alpha} \subset F_{1 a} \cap F_{\alpha} \subseteq F_{\alpha a}$.

In the following theorem, $g_{x}\left(e^{i t}\right)$ is $L^{1}$ continuous function of $x$ means,

$$
\left\|g_{x}\left(e^{i t}\right)-g_{y}\left(e^{i t}\right)\right\|_{L^{1}} \longrightarrow 0 \text { as } x \longrightarrow y,
$$


$g_{x}\left(e^{i t}\right)$ is $M^{+}$continuous, if

$$
\left\|\int_{x} g_{x}\left(e^{i t}\right) d \mu_{n}(x)-\int_{x} g_{x}\left(e^{i t}\right) d \mu(x)\right\|_{L^{1}} \longrightarrow 0,
$$

for any sequence $\left\{\mu_{n}\right\}$ of nonnegative Borel measures that are weak* convergent to $\mu$.

THEOREM 3.3. For a holomorphic self-map $\varphi$ of the unit disc $\mathbf{D}$ and $\alpha \geq 1$, if $C_{\varphi}$ is compact on $F_{\alpha}$, then $\left(C_{\varphi} \circ K_{x}^{\alpha}\right)(z) \in F_{\alpha a}$ and

$$
\left(C_{\varphi} \circ K_{x}^{\alpha}\right)(z)=\int_{-\pi}^{\pi} g_{x}\left(e^{i t}\right) K_{x}^{\alpha}(z) d t,
$$

where $g_{x}\left(e^{i t}\right)$ is a function of two variables $x$, with $|x|=1$ and $e^{i t},\left\|g_{x}\left(e^{i t}\right)\right\|_{L^{1}} \leq a<\infty$, $g_{x}\left(e^{i t}\right)$ is nonnegative, and $M^{+}$is a continuous function of $x$.

Proof. Assume that $C_{\varphi}$ is compact and let $\left\{f_{j}\right\}_{j=1}^{\infty}$ be a sequence of functions such that

$$
f_{j}(z)=K_{x}^{\alpha}\left(\rho_{j} z\right)=\frac{1}{\left(1-\rho_{j} \bar{x} z\right)^{\alpha}}
$$

where $0<\rho_{j}<1$ and $\lim _{j \rightarrow \infty} \rho_{j}=1$. Then, it is known from [2] that $f_{j}(z) \in F_{\alpha}$ for every $j$, and $\left\|f_{j}(z)\right\|_{F_{\alpha}}=1$. Furthermore, there exist $\mu_{j} \in \mathbf{M}$, such that $\left\|\mu_{j}\right\|=1, d \mu_{j}>0$, and

$$
\begin{aligned}
f_{j}(z) & =\frac{1}{\left(1-\rho_{j} \bar{x} z\right)^{\alpha}} \\
& =\int_{\mathrm{T}} K_{e^{i t}}^{\alpha}(z) d \mu_{j}\left(e^{i t}\right) \\
& =\int_{\mathrm{T}} \frac{1}{\left(1-e^{-i t} z\right)^{\alpha}} d \mu_{j}\left(e^{i t}\right) .
\end{aligned}
$$

Since $C_{\varphi}$ is compact on $F_{\alpha}$, then $\left(C_{\varphi} \circ f_{j}\right) \in F_{\alpha}$ and $\left\|C_{\varphi}\left(f_{j}\right)\right\| \leq\left\|C_{\varphi}\right\|\left\|f_{j}\right\|_{F_{\alpha}}=\left\|C_{\varphi}\right\|$ for all $j$. Furthermore, $C_{\varphi} \circ f_{j} \in H^{\infty}$, therefore using the previous result, we get that $\left(C_{\varphi} \circ f_{j}\right) \in H^{\infty} \cap F_{\alpha} \subset F_{\alpha a}$ for every $j$. Therefore, there exists $L^{1}$ a nonnegative function $g_{x}^{j}\left(e^{i t}\right)$ such that

$$
\begin{gathered}
d \mu_{j}\left(e^{i t}\right)=g_{x}^{j}\left(e^{i t}\right) d t, \quad\left\|g_{x}^{j}\right\|_{L^{1}} \leq\left\|C_{\varphi}\right\|, \\
\left(f_{j} \circ \varphi\right)(z)=K_{x}^{\alpha}\left(\rho_{j} \varphi\right)=\int_{-\pi}^{\pi} g_{x}^{j}\left(e^{i t}\right) K_{e^{i t}}^{\alpha}(z) d t .
\end{gathered}
$$

Now, because $F_{\alpha a}$ is closed and $C_{\varphi}$ is compact, the sequence $\left\{f_{j} \circ \varphi\right\}_{j=1}^{\infty}$ has a convergent subsequence $\left\{f_{j_{k}} \circ \varphi\right\}$ that converges to $\left(K_{x}^{\alpha} \circ \varphi\right)(z) \in F_{\alpha a}$. Therefore,

$$
\begin{aligned}
\lim _{k \rightarrow \infty}\left(f_{j_{k}} \circ \varphi\right)(z) & =\lim _{k \rightarrow \infty} K_{x}^{\alpha}\left(\rho_{j_{k}} \varphi\right) \\
& =\lim _{k \rightarrow \infty} \int_{-\pi}^{\pi} g_{x}^{j_{k}}\left(e^{i t}\right) K_{e^{i t}}^{\alpha}(z) d t \\
& =\int_{-\pi}^{\pi} g_{x}\left(e^{i t}\right) K_{e^{i t}}^{\alpha}(z) d t \\
& =\left(K_{x}^{\alpha} \circ \varphi\right)(z)=\frac{1}{(1-\bar{x} \varphi(z))^{\alpha}} \in F_{\alpha a},
\end{aligned}
$$


where the function $g_{x}\left(e^{i t}\right)$ is an $L^{1}$ nonnegative continuous function of $x$, and $\left\|g_{x}\right\|_{L^{1}}$ $\leq\left\|C_{\varphi}\right\|$. The continuity of $g_{x}\left(e^{i t}\right)$ in $M^{+}$follows from the compactness and the fact that if $f(z)=\int_{\mathrm{T}} K_{x}^{\alpha}(z) d \mu(x)$, with $d \mu(x)>0$, then $\|f\|_{F_{\alpha}}=\|\mu\|$.

This concludes the proof.

COROLLARY 3.4. Let $g_{x}\left(e^{i t}\right)$ be as in Theorem 3.3. Then, for $h(x) \in \overline{H_{0}^{1}}$, the operator $\int g_{x}\left(e^{i t}\right) h(x) d x=u\left(e^{i t}\right) \in \overline{H_{0}^{1}}$ is bounded on $\overline{H_{0}^{1}}$.

Proof. For the operator to be well defined, $\int\left(h(x) d x /(1-\bar{x} \varphi(z))^{\alpha}\right)=0$ for all $h(x) \in \overline{H_{0}^{1}}$. Hence, $\int g_{x}\left(e^{i t}\right) h(x) d x=u\left(e^{i t}\right) \in \overline{H_{0}^{1}}$.

In the opposite direction, we have the following lemma.

LEMMA 3.5. Let $g_{x}\left(e^{i t}\right)$ be a nonnegative $M^{+}$continuous function such that $\left\|g_{x}\right\|_{L^{1}} \leq$ $a<\infty$ and $g_{x}\left(e^{i t}\right)$ defines a bounded operator on $\overline{H_{0}^{1}}$. If $f(z)=\int\left(1 /(1-\bar{x} z)^{\alpha}\right) d \mu(x)$, let $L$ be the operator given by

$$
L[f(z)]=\iint \frac{g_{x}\left(e^{i t}\right)}{\left(1-e^{-i t} z\right)^{\alpha}} d t d \mu(x),
$$

then $L$ is compact operator on $F_{\alpha}, \alpha \geq 1$.

Proof. First, note that the condition that $g_{x}\left(e^{i t}\right)$ defines a bounded operator on $\overline{H_{0}^{1}}$ implies that the $L$ operator is a well-defined function on $\mathbf{F}_{\alpha}$. Let $\left\{f_{n}(z)\right\}$ be a bounded sequence in $F_{\alpha}$ and let $\left\{\mu_{n}\right\}$ be the corresponding norm bounded sequence of measures in $\mathbf{M}$. Since every norm bounded sequence of measures in $\mathbf{M}$ has a weak* convergent subsequence, let $\left\{\mu_{n}\right\}$ be such subsequence that is convergent to $\mu \in \mathbf{M}$. We want to show that $\left\{L\left(f_{n}\right)\right\}$ has a convergent subsequence in $F_{\alpha}$.

First, we assume that $d \mu_{n}(x)>0$ for all $n$, and let $w_{n}(t)=\int g_{x}\left(e^{i t}\right) d \mu_{n}(x)$ and $w(t)=\int g_{x}\left(e^{i t}\right) d \mu(x)$, then, as $g_{x}\left(e^{i t}\right)$ is an $M^{+}$continuous function, we have $w_{n}(t)$, $w(t) \in L^{1}$ for all $n$, and $w_{n}(t) \rightarrow w(t)$ in $L^{1}$. Now, since $g_{x}\left(e^{i t}\right)$ is a nonnegative continuous function in $x$ and $\left\{\mu_{n}\right\}$ is weak* convergent to $\mu$, then

$$
\begin{aligned}
L\left(f_{n}(z)\right) & =\iint \frac{g_{x}\left(e^{i t}\right) d(t)}{\left(1-e^{-i t} z\right)^{\alpha}} d \mu_{n}(x)=\int \frac{w_{n}(t)}{\left(1-e^{-i t} z\right)^{\alpha}} d t, \\
L(f(z)) & =\iint \frac{g_{x}\left(e^{i t}\right) d(t)}{\left(1-e^{-i t} z\right)^{\alpha}} d \mu(x)=\int \frac{w(t)}{\left(1-e^{-i t} z\right)^{\alpha}} d t .
\end{aligned}
$$

Furthermore, because $w_{n}(t)$ is nonnegative, then

$$
\begin{gathered}
\left\|L\left(f_{n}\right)\right\|_{F_{\alpha}}=\left\|w_{n}\right\|_{L^{1}}, \\
\|L(f)\|_{F_{\alpha}}=\|w\|_{L^{1}} .
\end{gathered}
$$

Now, since $\left\|w_{n}-w\right\|_{L^{1}} \rightarrow 0$, then $\left\|L\left(f_{n}\right)-L(f)\right\|_{F_{\alpha}} \rightarrow 0$, which shows that $\left\{L\left(f_{n}\right)\right\}$ has a convergent subsequence in $F_{\alpha}$ and thus $L$ is a compact operator for the case where $\mu$ is a positive measure.

In the case where $\mu$ is complex measure, we write

$$
d \mu_{n}(x)=\left(d \mu_{n}^{1}(x)-d \mu_{n}^{2}(x)\right)+i\left(d \mu_{n}^{3}(x)-d \mu_{n}^{4}(x)\right),
$$


where each $d \mu_{n}^{j}(x)>0$, and define $w_{n}^{j}(t)=\int g_{x}\left(e^{i t}\right) d \mu_{n}^{j}(x)$, then

$$
w_{n}(t)=\int g_{x}\left(e^{i t}\right) d \mu_{n}(x)=\left(w_{n}^{1}(t)-w_{n}^{2}(t)\right)+i\left(w_{n}^{3}(t)-w_{n}^{4}(t)\right)
$$

Using an argument similar to the one above, we get that $w_{n}^{j}(t), w^{j}(t) \in L^{1}$, and $\left\|w_{n}^{j}-w^{j}\right\|_{L^{1}} \rightarrow 0$. Consequently, $\left\|w_{n}-w\right\|_{L^{1}} \rightarrow 0$, where $w(t)=\left(w^{1}(t)-w^{2}(t)\right)+$ $i\left(w^{3}(t)-w^{4}(t)\right)=\int g_{x}\left(e^{i t}\right) d \mu(x)$.

Hence, $\left\|L\left(f_{n}\right)-L(f)\right\|_{F_{\alpha}} \leq\left\|w_{n}-w\right\|_{L^{1}} \rightarrow 0$.

Finally, we conclude that the operator is compact.

The following is the converse of Theorem 3.3.

THEOREM 3.6. For a holomorphic self-map $\varphi$ of the unit disc $\mathbf{D}$, if

$$
\left(K_{x}^{\alpha} \circ \varphi\right)(z)=\frac{1}{(1-\bar{x} \varphi(z))^{\alpha}}=\int \frac{g_{x}\left(e^{i t}\right)}{\left(1-e^{-i t} z\right)^{\alpha}} d t, \quad g_{x} \in L^{1}
$$

is nonnegative, $\left\|g_{x}\right\|_{L^{1}} \leq a<\infty$ for all $x \in \mathbf{T}$ and $g_{x}$ is an $M^{+}$continuous function, then $C_{\varphi}$ is compact on $F_{\alpha}$.

Proof. We want to show that $C_{\varphi}$ is compact on $F_{\alpha}$. Let $f(z) \in F_{\alpha}$. Then, there exists a measure $\mu$ in $\mathbf{M}$ such that for every $z$ in $D$,

$$
f(z)=\int \frac{1}{(1-\bar{x} z)^{\alpha}} d \mu(x)
$$

Using the assumption of the theorem, we get that

$$
(f \circ \varphi)(z)=\int \frac{1}{(1-\bar{x} \varphi(z))^{\alpha}} d \mu_{n}(x)=\iint \frac{g_{x}\left(e^{i t}\right)}{\left(1-e^{-i t} z\right)^{\alpha}} d t d \mu_{n}(x),
$$

which by the previous lemma was shown to be compact on $F_{\alpha}$.

Now, we give some examples.

Corollary 3.7. Let $\varphi \in H(\mathbf{D})$, with $\|\varphi\|_{\infty}<1$. Then, $C_{\varphi}$ is compact on $F_{\alpha}, \alpha \geq 1$.

Proof. $\left(C_{\varphi} \circ K_{x}^{\alpha}\right)(z)=1 /(1-\bar{x} \varphi(z))^{\alpha} \in H^{\infty} \cap F_{\alpha} \subset F_{\alpha a}$ and is subordinate to $1 /(1-z)^{\alpha}$, hence,

$$
\left(C_{\varphi} \circ K_{x}^{\alpha}\right)(z)=\int K_{x}^{\alpha}(z) g_{x}\left(e^{i t}\right) d t
$$

with $g_{x}\left(e^{i t}\right) \geq 0$ and since $1=\left(C_{\varphi} \circ K_{x}^{\alpha}\right)(0)=\int g_{x}\left(e^{i t}\right) d t$, we get that $\left\|g_{x}\left(e^{i t}\right)\right\|_{1}=1$.

REMARK 3.8. In fact, one can show that $C_{\varphi}$, as in the above corollary, is compact from $F_{\alpha}, \alpha \geq 1$, into $F_{1}$. In other words, a contraction. 
COROLlary 3.9. If $C_{\varphi}$ is compact on $F_{\alpha}, \alpha \geq 1$, and $\lim _{r \rightarrow 1}\left|\varphi\left(r e^{i \theta}\right)\right|=1$, then $\left|1 / \varphi^{\prime}\left(e^{i \theta}\right)\right|=0$.

Proof. If $C_{\varphi}$ is compact, then

$$
\left(C_{\varphi} \circ K_{x}^{\alpha}\right)(z)=\int K_{x}^{\alpha}(z) g_{x}\left(e^{i t}\right) d t .
$$

Hence, if $z=e^{i \theta}$ and $\varphi\left(e^{i \theta}\right)=x$, then

$$
\lim _{r \rightarrow 1} \frac{\left(e^{i \theta}-r e^{i \theta}\right)^{\alpha}}{\left(1-\bar{x} \varphi\left(r e^{i \theta}\right)\right)^{\alpha}}=0 .
$$

COROLLARY 3.10. If $C_{\varphi} \in K\left(F_{\alpha}, F_{\alpha}\right)$ for $\alpha \geq 1$, then $C_{\varphi}$ is a contraction.

4. Miscellaneous results. We first start by giving another characterization of compactness on $F_{\alpha}$.

LEMmA 4.1. Let $\varphi \in C\left(F_{\alpha}, F_{\alpha}\right), \alpha>0$, then $\varphi \in K\left(F_{\alpha}, F_{\alpha}\right)$ if and only if for any bounded sequence $\left(f_{n}\right)$ in $F_{\alpha}$, with $f_{n} \rightarrow 0$ uniformly on compact subsets of $\mathbf{D}$ as $n \rightarrow \infty$, $\left\|C_{\varphi}\left(f_{n}\right)\right\|_{F_{\alpha}} \rightarrow 0$ as $n \rightarrow \infty$.

Proof. Suppose $C_{\varphi} \in K\left(F_{\alpha}, F_{\alpha}\right)$ and let $\left(f_{n}\right)$ be a bounded sequence $\left(f_{n}\right)$ in $F_{\alpha}$ with $\lim _{n \rightarrow \infty} f_{n} \rightarrow 0$ uniformly on compact subsets of $\mathbf{D}$. If the conclusion is false, then there exists an $\epsilon>0$ and a subsequence $n_{1}<n_{2}<n_{3}<\cdots$ such that

$$
\left\|C_{\varphi}\left(f_{n_{j}}\right)\right\|_{F_{\alpha}} \geq \epsilon, \quad \forall j=1,2,3, \ldots
$$

Since $\left(f_{n}\right)$ is bounded and $C_{\varphi}$ is compact, one can find another subsequence $n_{j_{1}}<$ $n_{j_{2}}<n_{j_{3}}<\cdots$ and $f$ in $F_{\alpha}$ such that

$$
\lim _{k \rightarrow \infty}\left\|C_{\varphi}\left(f_{n_{j_{k}}}\right)-f\right\|_{F_{\alpha}}=0 .
$$

Since point functional evaluations are continuous in $F_{\alpha}$, then for any $z \in \mathbf{D}$ there exists $A>0$ such that

$$
\left|\left(C_{\varphi}\left(f_{n_{j_{k}}}\right)-f\right)(z)\right| \leq A\left\|C_{\varphi}\left(f_{n_{j_{k}}}\right)-f\right\|_{F_{\alpha}} \rightarrow 0 \text { as } k \rightarrow \infty .
$$

Hence,

$$
\lim _{k \rightarrow \infty}\left[C_{\varphi}\left(f_{n_{j_{k}}}\right)-f\right] \rightarrow 0
$$

uniformly on compact subsets of D. Moreover, since $f_{n_{j_{k}}} \rightarrow 0$ uniformly on compact subsets of $\mathbf{D}$, then $f=0$, that is, $C_{\varphi}\left(f_{n_{j_{k}}}\right) \rightarrow 0$ on compact subsets of $F_{\alpha}$. Hence,

$$
\lim _{k \rightarrow \infty}\left\|C_{\varphi}\left(f_{n_{j_{k}}}\right)\right\|_{F_{\alpha}}=0,
$$


which contradicts our assumption. Thus, we must have

$$
\lim _{n \rightarrow \infty}\left\|C_{\varphi}\left(f_{n}\right)\right\|_{F_{\alpha}}=0 .
$$

Conversely, let $\left(f_{n}\right)$ be a bounded sequence in the closed unit ball of $F_{\alpha}$. We want to show that $C_{\varphi}\left(f_{n}\right)$ has a norm convergent subsequence. The closed unit ball of $F_{\alpha}$ is a compact subset of $F_{\alpha}$ in the topology of uniform convergence on the compact subsets of $\mathbf{D}$. Therefore, there is a subsequence $\left(f_{n_{k}}\right)$ such that

$$
f_{n_{k}} \rightarrow f
$$

uniformly on compact subsets of $D$. Hence, by hypothesis,

$$
\left\|C_{\varphi}\left(f_{n_{k}}\right)-C_{\varphi}(f)\right\|_{F_{\alpha}} \longrightarrow 0 \text { as } k \longrightarrow \infty,
$$

which completes the proof.

Proposition 4.2. If $C_{\varphi} \in C\left(F_{\alpha}, F_{\alpha}\right)$, then $C_{\varphi} \in K\left(F_{\alpha}, F_{\beta}\right)$ for all $\beta>\alpha>0$.

Proof. Let $\left(f_{n}\right)$ be a bounded sequence in the closed unit ball of $F_{\alpha}$. Then, $\left(f_{n} \circ \varphi\right)$ is bounded in $F_{\alpha}$ and since the inclusion map $i: F_{\alpha} \rightarrow F_{\beta}$ is compact, $\left(f_{n} \circ \varphi\right)$ has a convergent subsequence in $F_{\beta}$.

Proposition 4.3. $C_{\varphi}(f)=(f \circ \varphi)$ is compact on $F_{\alpha}$ if and only if the operator $\varphi^{\prime} C_{\varphi}(g)=\varphi^{\prime}(g \circ \varphi)$ is compact on $F_{\alpha+1}$.

Proof. Suppose that $C_{\varphi}(f)=(f \circ \varphi)$ is compact on $F_{\alpha}$. It is known from [4] that $\varphi^{\prime} C_{\varphi}(g)=\varphi^{\prime}(g \circ \varphi)$ is bounded on $F_{\alpha+1}$. Let $\left(g_{n}\right)$ be a bounded sequence in $F_{\alpha+1}$, with $g_{n} \rightarrow 0$ uniformly on compact subsets of $\mathbf{D}$ as $n \rightarrow \infty$. We want to show that $\lim _{n \rightarrow \infty}\left\|\varphi^{\prime}\left(g_{n} \circ \varphi\right)\right\|_{F_{\alpha+1}}=0$. Let $\left(f_{n}\right)$ be the sequence defined by $f_{n}(z)=\int_{0}^{z} g_{n}(w) d w$. Then, $f_{n} \in F_{\alpha}$ and $\left\|f_{n}\right\|_{F_{\alpha}} \leq(2 / \alpha)\left\|g_{n}\right\|_{F_{\alpha+1}}$, thus $\left(f_{n}\right)$ is a bounded sequence in $F_{\alpha}$. Furthermore, using the Lebesgue dominated convergence theorem we get that $f_{n} \rightarrow 0$ uniformly on compact subsets of D. Thus,

$$
\begin{aligned}
\left\|\varphi^{\prime}\left(g_{n} \circ \varphi\right)\right\|_{F_{\alpha+1}} & =\left\|\varphi^{\prime}\left(f_{n}^{\prime} \circ \varphi\right)\right\|_{F_{\alpha+1}} \\
& =\left\|\left(f_{n} \circ \varphi\right)^{\prime}\right\|_{F_{\alpha+1}} \\
& \leq \alpha\left\|\left(f_{n} \circ \varphi\right)\right\|_{F_{\alpha}} \longrightarrow 0 \text { as } n \longrightarrow \infty,
\end{aligned}
$$

which shows that $\varphi^{\prime} C_{\varphi}(g)=\varphi^{\prime}(g \circ \varphi)$ is compact on $F_{\alpha+1}$.

Conversely, assume that $\varphi^{\prime} C_{\varphi}(g)=\varphi^{\prime}(g \circ \varphi)$ is compact on $F_{\alpha+1}$. Then, in particular, $\varphi^{\prime} C_{\varphi}\left(f^{\prime}\right)=\varphi^{\prime}\left(f^{\prime} \circ \varphi\right)=(f \circ \varphi)^{\prime}$ is a compact for every $f \in F_{\alpha}$. Now, since $\|(f \circ \varphi)\|_{F_{\alpha}} \leq$ $(2 / \alpha)\left\|(f \circ \varphi)^{\prime}\right\|_{F_{\alpha+1}}$. Let $\left(f_{n}\right)$ be a bounded sequence in $F_{\alpha}$ with $f_{n} \rightarrow 0$ uniformly on compact subsets of $\mathbf{D}$ as $n \rightarrow \infty$. We want to show that $\lim _{n \rightarrow \infty}\left\|\left(f_{n} \circ \varphi\right)\right\|_{F_{\alpha}}=0$. Since any bounded sequence of $F_{\alpha}$ is also a bounded sequence of $F_{\alpha+1}$, then $\left\|\left(f_{n} \circ \varphi\right)\right\|_{F_{\alpha}} \leq$ $(2 / \alpha)\left\|\left(f_{n} \circ \varphi\right)^{\prime}\right\|_{F_{\alpha+1}} \rightarrow 0$ as $n \rightarrow \infty$ and the proof is complete.

ACKNOWLEDGMENT. The authors would like to thank the referee for his many helpful suggestions and comments. 


\section{REFERENCES}

[1] J. A. Cima and A. Matheson, Cauchy transforms and composition operators, Illinois J. Math. 42 (1998), no. 1, 58-69.

[2] D. J. Hallenbeck, T. H. MacGregor, and K. Samotij, Fractional Cauchy transforms, inner functions and multipliers, Proc. London Math. Soc. (3) 72 (1996), no. 1, 157-187.

[3] D. J. Hallenbeck and K. Samotij, On Cauchy integrals of logarithmic potentials and their multipliers, J. Math. Anal. Appl. 174 (1993), no. 2, 614-634.

[4] R. A. Hibschweiler, Composition operators on spaces of Cauchy transforms, Studies on Composition Operators (Laramie, Wyo, 1996), Contemp. Math., vol. 213, American Mathematical Society, Rhode Island, 1998, pp. 57-63.

[5] R. A. Hibschweiler and T. H. MacGregor, Closure properties of families of Cauchy-Stieltjes transforms, Proc. Amer. Math. Soc. 105 (1989), no. 3, 615-621.

[6] R. A. Hibschweiler and E. Nordgren, Cauchy transforms of measures and weighted shift operators on the disc algebra, Rocky Mountain J. Math. 26 (1996), no. 2, 627-654.

[7] T. H. MacGregor, Analytic and univalent functions with integral representations involving complex measures, Indiana Univ. Math. J. 36 (1987), no. 1, 109-130.

Yusuf Abu Muhanna: Department of Mathematics, American University of Sharjah, P.O. Box 26666, Sharjah, United Arab Emirates

E-mail address: ymuhanna@aus . ac . ae

El-Bachir Yallaoui: College of Arts and Sciences, Abu Dhabi University, P.O. Box 1790, Al Ain, United Arab Emirates

E-mail address: e1bachir.ya11aoui@adu.ae 


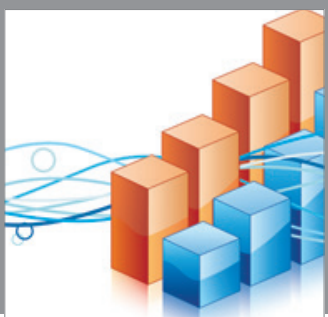

Advances in

Operations Research

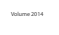

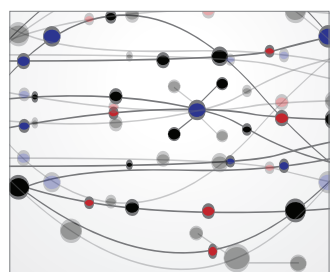

\section{The Scientific} World Journal
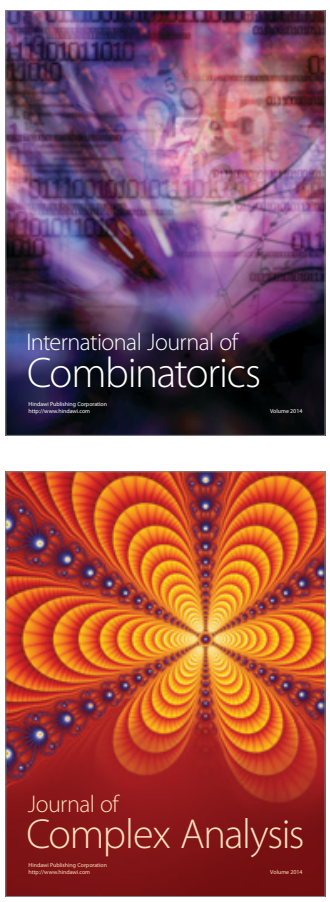

International Journal of

Mathematics and

Mathematical

Sciences
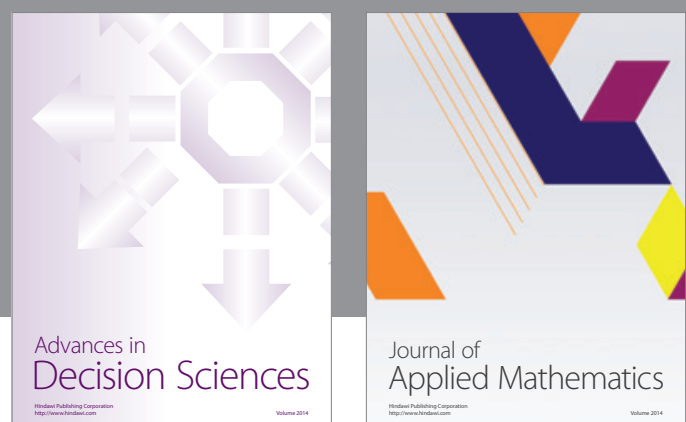

Journal of

Applied Mathematics
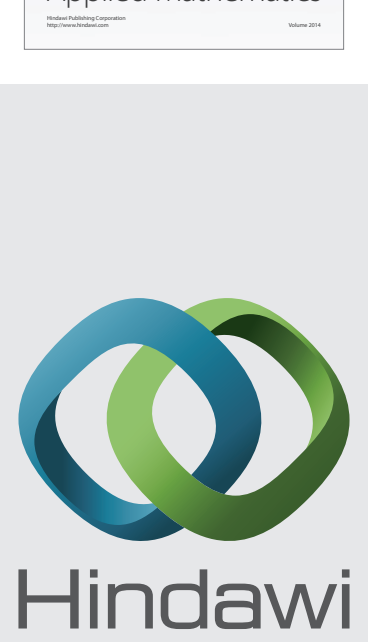

Submit your manuscripts at http://www.hindawi.com
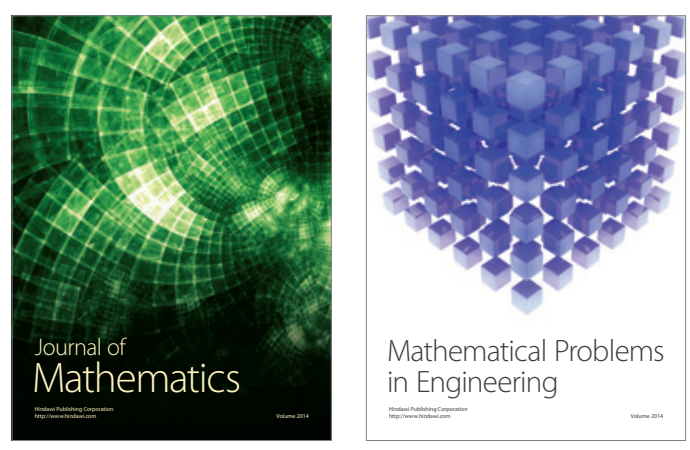

Mathematical Problems in Engineering
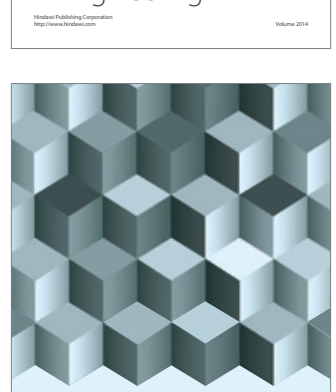

Journal of

Function Spaces
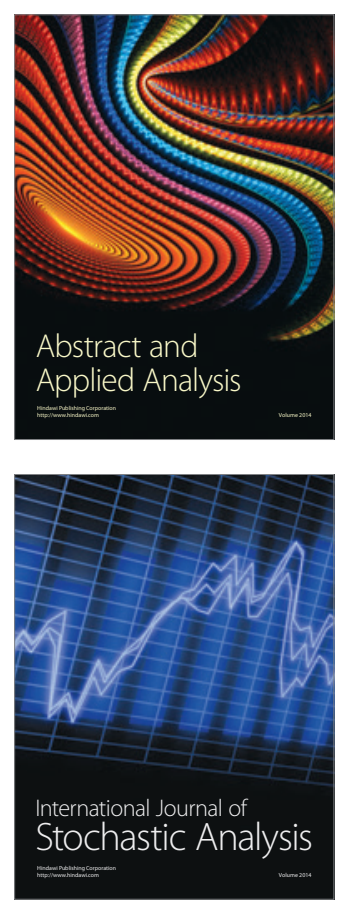

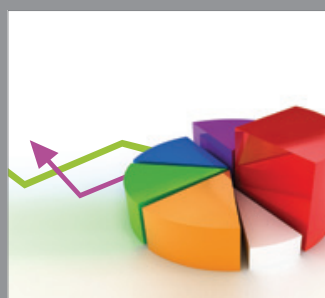

ournal of

Probability and Statistics

Promensencen
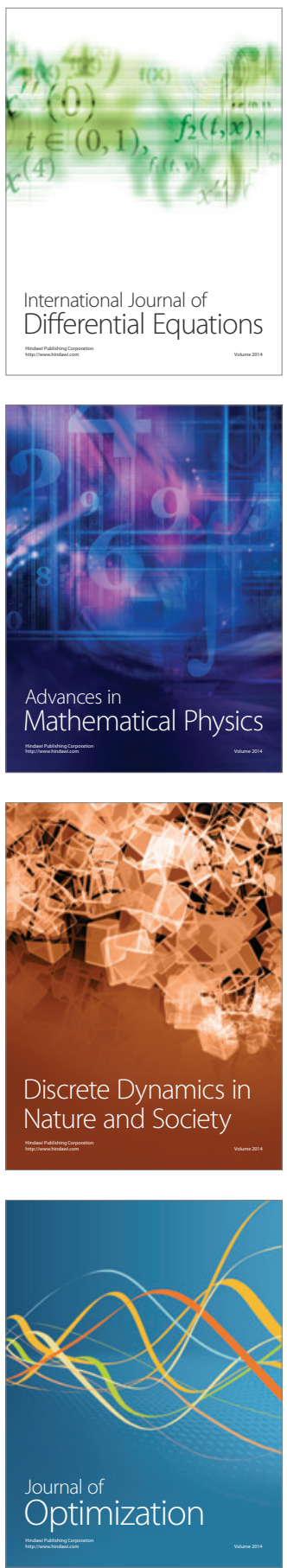